\title{
Acupoint Therapy on Diabetes Mellitus and Its Common Chronic Complications: A Review of Its Mechanisms
}

\author{
Yiyi Feng, Yuchen Fang, Yiqin Wang $\mathbb{D}$, and Yiming Hao $\mathbb{D}$ \\ Shanghai Key Laboratory of Health Identification and Assessment/Laboratory of TCM Four Diagnostic Information, \\ Shanghai University of Traditional Chinese Medicine, Shanghai, 201203, China
}

Correspondence should be addressed to Yiming Hao; hymjj888@163.com

Received 25 May 2018; Revised 22 September 2018; Accepted 8 October 2018; Published 22 October 2018

Academic Editor: Swaran J. S. Flora

Copyright (C) 2018 Yiyi Feng et al. This is an open access article distributed under the Creative Commons Attribution License, which permits unrestricted use, distribution, and reproduction in any medium, provided the original work is properly cited.

\begin{abstract}
Acupoint therapy is one of the therapeutic means in Traditional Chinese Medicine (TCM) concerning acupoints and meridians, including manual acupuncture, electroacupuncture, moxibustion, external application, acupoint injection, and catgut embedding. In the treatment of diabetes and its common chronic complications, acupoint therapy has proved to have specific curative effect and notable advantages. Single or combined with western medicine, it has superior efficacy and less side effects than western medicine alone. Studying its mechanism can provide experimental basis for clinical treatment. Relevant researches in the recent 5 years mainly focused on the mechanism of electroacupuncture, point injection, catgut embedding, etc. in the treatment of diabetes and common diabetic complications such as neuropathy, nephropathy, and hepatopathy. The possible theories involve the regulation of nerve conduction, signal pathways, hormone level, protein expression, oxidative stress level, structure restoration, etc. The most studied acupoints are Zusanli (ST36), Shenshu (BL23), Sanyinjiao (SP6), Yishu (EX-B3), and Zhongwan (CV12). However, most of the studies have been based on diabetes model rats rather than clinical trials. Moreover, the mechanism of acupoint therapy treating other chronic complications like diabetic retinopathy and that of other effective methods like pressing ear with beans, auricular points plaster therapy, and external application remain unclear. Therefore, this aspect still awaits further research.
\end{abstract}

\section{Introduction}

Diabetes mellitus (DM) is a ubiquitous metabolic disease which severely threatens the health and even the survival of humankind. Chronic hyperglycaemia is very likely to result in vascular damage, which will lead to a series of diabetic complications in heart, liver, stomach, kidney, muscle, peripheral nerve, etc. According to Global Report on Diabetes [1], diabetic complications may cause heart attack, stroke, blindness, renal failure, and lower limb amputation which have deadly consequences; in 2012, DM alone brought about the death of 1.5 million patients while its complications devitalized another 2.2 million patients. Acupoint therapy of Traditional Chinese Medicine (TCM) may be a way out. It is one of the therapeutic means in TCM concerning acupoints and meridians, including manual acupuncture, electroacupuncture, moxibustion, external application, acupoint injection, and catgut embedding. Acupoint therapy has proved to have specific curative effect and notable advantages in the treatment of DM and its chronic complications. Single or combined with Western medicine, it has more superior efficacy and less side effects than Western medicine alone. Many researches have shown that methods like electroacupuncture, acupoint injection, and catgut embedding can effectively decrease blood glucose, enhance insulin sensitivity, alleviate symptoms of various complications, and even prevent diabetes in specific populations [2]. Studying its mechanism will be conducive to its clinical application, thus benefiting diabetic patients worldwide. Our study reviewed relevant researches in the recent 5 years regarding the latest progress of the mechanism study of acupoint therapy in the treatment of DM and its common chronic complications. Currently, most of the studies are experiments on streptozotocin- (STZ-) induced Type 2 Diabetes Mellitus (T2DM) model rats.

\section{The Mechanism Study of Hyperglycaemia Acupoint Treatment}

Hyperglycaemia, which resulted from insulin secretion dysfunction or impairment of insulin biological function, is 
one of the most fundamental characteristics of diabetes mellitus. Acupoint therapy has been repeatedly confirmed to have evident effects on decreasing blood glucose. The existing studies have found that the regulation of related nerve excitement, protein expression, and signaling pathways that strengthens insulin sensitivity plays an important role in the treatment.

The endocrine system and the nervous system coregulate the metabolic activities in our bodies and they influence each other significantly. Yishu (EX-B3) is located below the $8^{\text {th }}$ spinous process of thoracic vertebrae, where there is part of $\mathrm{T}_{8}$ that innervates the pancreas. Electroacupuncture on T2DM rats at point Yishu (EX-B3) helps to reduce blood glucose and protect the shape of islets, which is consistent with the theory of innervation of neural segment [3]. The theory describes the neural connection between body surface and internal organs. The main functions of a certain acupoint are focused on the organs innervated by neural system adjacent to the acupoint. As long as a group of acupoints are located in the same neural segment, their functions are similar to each other even if they are not from the same meridian [4]. Obviously, the regulation of Yishu (EX-B3) on the pancreas is conformable to this theory. Besides, the hypothalamic-pituitary-adrenal axis (HPA), as an important part of neuro-endocrine system, also exerts influence on the insulin secretion. Electroacupuncture at Yishu (EX-B3) is demonstrated to decrease the level of adrenal cortical hormone (CORT) and reduce HPA hyperfunction, thus ameliorating endocrine dyscrasia and improving physical as well as psychological health status of rats to some extent [5].

Another important initial factor of T2DM is insulin resistance. One of the characteristic indexes of insulin resistance is high fasting insulin (FINS) level due to the patients' poor insulin sensitivity and low efficiency of uptaking and utilizing glucose. Electroacupuncture at Zusanli (ST36) and Shenshu (BL23) is reported to reduce the level of FINS and protect islet $B$ cell morphology by enhancing mRNA expression of GLUT2 and GCK [6]. GLUT2 is an efficient carrier for glucose and GCK is an enzyme that facilitates phosphorylation of glucose, both of which function together as "glucose sensor" and respond to fluctuations of glucose level. Enhancement of them leads to improvement of insulin sensitivity and ameliorate insulin resistance. Manual acupuncture on Quchi (LI11), Hegu (LI4), Zusanli (ST36), Xuehai (SP10), Fenglong (ST40), Yinlingquan (SP9), Diji (SP8), Sanyinjiao (SP6), and Taichong (LR3) with "spleenstomach harmonizing" technique can also reduce insulin resistance. "Spleen-stomach harmonizing" technique is created by Doctor Zhang Zhilong with a series of operations on the acupoints mentioned mainly for curing Type 2 diabetes mellitus and its complications. The study shows it possibly reduces insulin resistance by increasing the gene expression level of IRS-1, IRS-2, and GLUT4 in muscular tissue which suggests an activation of the signal transduction pathway of the phosphatidylinositol 3-kinase (PI3K)/Akt [7]. More studies have confirmed the effect of electroacupuncture on the PI3K/Akt signal transduction pathway. In this pathway, the activity of PI3K of insulin resistant rats is usually inhibited. Normally, its regulatory subunit p85 adjusts the catalytic activity of another subunit p110 and realizes the functions of insulin. However, exceedingly expressed p $85 \alpha$ competitively inhibit the binding of p85-p110 and its downstream molecule IRS-1 so that the function of PI3K is suppressed. A study found that electroacupuncture at Zusanli (ST36) and Quchi (LI11) decreases the expression of PI3K-p85 to ameliorate the insulin resistance of model rats [8]. A more explicit research has shown that electroacupuncture at Neiguan (PC6), Zusanli (ST36), Sanyinjiao (SP6), and Shenshu (BL23) restores the level of insulin signaling related molecules like IRS-1, IRS-2, Akt2, aPKC $\zeta$, and GLUT4 to normal and reversed the increased PI3K-p85 $\alpha$ level [9]. In this way, metabolic activities like glycogen synthesis are improved via PI3K/Akt signaling pathway. In addition, the improvement of insulin resistance can reverse the pathological changes of vascular endothelium. Vascular endothelial dysfunction is the pathological basis and early stages of atherosclerosis, which is inseparable and forms a vicious cycle with the occurrence of insulin resistance [10]. It turns out to be another result of PI3K/Akt signalling pathway regulation. Researchers have studied the effect of electroacupuncture at Shenshu (BL23), Neiguan (PC6), Zusanli (ST36), and Sanyinjiao (SP6) on T2DM rats which demonstrates an increase in the expression of IRS-1, PI3K, Akt2, and eNOS in vascular endothelium. This signal transduction pathway is the downstream of insulin in vascular endothelium cells. When insulin sensitivity is enhanced, the signalling pathway can be better activated and promote the production of NO. In this way, vascular endothelial dysfunction is mitigated, and vasodilation can exert positive counteraction on glucose utilization [11].

\section{The Mechanism Study of Diabetic Neuropathy Acupoint Treatment}

Diabetic neuropathy is a kind of peripheral or central neuropathy caused by diabetic metabolic disturbance and angiopathy. The percent of complicated neuropathy ranges from $60 \%$ to $80 \%$ and its mortality rate reaches $20 \%$ in ten years from its occurrence [12]. It includes a wide variety of diseases and acupoint therapy interferes them in different mechanisms as follows.

3.1. Diabetic Peripheral Neuropathy (DPN). The incidence of peripheral neuropathy in T2DM patients reached more than $60 \%$ and among them only approximately $53 \%$ of DPN patients survive after 3 years of its occurence $[13,14]$. It may decrease sensory nerve conduction velocity (SNCV) and impair sensation or movement. Acupoint therapy can effectively increase SNCV and alleviate subjective symptoms. The mechanism involves upregulating the positive factors and downregulating the negative ones. For instance, electroacupuncture and acupoint injection with mecobalamin at Zusanli (ST36) and Shenshu (BL23) are demonstrated to increase the expression of nerve growth factor (NGF) and its receptor TrkA [15]. Likewise, catgut implantation at Pishu (BL20), Shenshu (BL23), and Zusanli (ST36) combined with taking herbal remedy, TCM prescription "Tangtong drink," enhances the content of NGF plus lower that of serum transforming growth factor- $\beta 1$ (TGF- $\beta 1$ ) [16]. They 
both promote the regeneration of nerves and repair of nerve injuries. Other positive regulative factors that are reported to be raised by acupuncture or acupoint injection includes superoxide dismutase (SOD) expression [17], content of nitric oxide (NO) in the serum [18]. SOD is an important antioxidant defense of living cells exposed to oxygen and can have positive effect on alleviating cell damage. Low-level of NO, as previously mentioned, is related to vascular endothelium dysfunction that can lead to nerve hypoxia and ischemia. What is more, $\mathrm{NO}$ is an important inhibitory neurotransmitter and the lack of it can cause disorders of neural functions. The increase of these molecules apparently exerts benign effects on neuropathy patients. On the contrary, researchers who treated DPN patients with salvia injections at Zusanli (ST36) combined with lipoid acid intravenous injection found that the expression of high-sensitivity C-reactive protein (hs$\mathrm{CRP}$ ) and the content of malondialdehyde (MDA) were significantly decreased [17]. Another similar study on lowfrequent electroacupuncture at Zhongwan (CV12), Mingmen (DU4), and Zusanli (ST36) combined with mecobalamin injection has shown the same result of reducing hs-CRP as well as homocysteine (Hcy) [19]. High level of hs-CRP, MDA, and Hcy mentioned above is correlated to causing or aggravating neuropathy, so their decrease protects the structure of neurons.

Another common DPN is diabetic gastroparesis. This disease usually results from vagus nerve lesion, which mainly manifests as delayed gastric emptying, decreased gastrointestinal motility and weakened gastric electrical activity with an incidence of 50\%-76\% [20]. Recent studies have focused on the interstitial cells of Cajal (ICCs) in the gastric antrum, gastrointestinal nervous system and hormones, etc. [21] Firstly, it was observed that the ultrastructure of ICCs and its pacing function was restored, and cell apoptosis remarkably reduced after the treatment of electroacupuncture at points of Zusanli (ST36) and Zhongwan (CV12). This is possibly concerned with the upregulation of stem cell factors (SCF) mRNA and neuronal nitric oxide synthase (nNOS) mRNA by mSCF/KIT-ETV1 signaling pathway [22-26]. Secondly, related hormones as important regulators of gastrointestinal functions and motility are affected directly or indirectly. For example, the levels of motilin, a gastrointestinal motility enhancer, and gastrin, the high-level of which has inhibitory effect on gastric movement, are obviously elevated in gastroparesis patients. The secretion of them will be inhibited by abdominal acupuncture at Zhongwan (CV12), Xiawan (RN10), Qihai (BL24), Guanyuan (BL26), Tianshu (ST25), Daheng (SP15), Huaroumen (ST24), and Wailing (ST26), as well as point injection of Vitamin $B_{12}$ or mecobalamin at Zusanli (ST36), the effect of which is superior to using western medicine like mosapride alone [20, 27]. Studies of electroacupuncture at Zusanli (ST36), Liangmen (ST21), and Sanyinjiao (SP6) also revealed a growth in endothelial nitric oxide synthase (eNOS) mRNA, insulin, and its receptor (IR), as well as a cut-down on AT II mRNA, insulin-like growth factor-1 (IGF-1), and its receptor (IGF-1 R) in the gastric antrum. Among these molecules, the binding of insulin and IR protects ICCs and the expression of eNOS mRNA can lead to more abundant content of NO which can ameliorate vascular endothelial dysfunction as mentioned. AT II, on the other hand, play its role by binding with AT I receptor and AT II receptor, the former of which is found increased in gastric tissue of DGP rats. Likewise, IGF-1 and its receptor are found significantly higher in DGP rats so the cut-down of them might suggest an action mechanism of electroacupuncture [28-30]. As a matter of fact, the restoration of Cajal interstitial cells, mediation of hormones, and nervous system supplement each other. With the treatment of needling at specific points, the amount of Cajal interstitial cells increases and gastric slow wave is regulated, thus alleviating gastric myoelectric arrhythmias, promoting signal transduction between nervous system and smooth muscle, and eventually normalizing gastric motor [31]. However, there still remain some mysteries unsolved. Researchers have paid attention to the modulation of ghrelin and its role in strengthening gastric motility. Some concluded that electroapuncture at Zusanli, Liangmen (ST21), and Sanyinjiao (SP6) contributes to an increase in ghrelin and growth hormone secretagogue receptor (GHSR) mRNA while another group had a contradictive result under the similar experimental conditions that the level of ghrelin mRNA declines after the treatment [32-35]. Whether it is because of human error or an undiscovered underlying mechanism needs further investigation. One conjecture of our group is that some unknown factors made the pathology of diabetic gastroparesis of the model rats in two groups different. One decreases the ghrelin while the other increases it. Acupuncture played a mediative role, respectively, in both cases and had the tendency to balance the body status.

3.2. Diabetic Cardiac Autonomic Neuropathy. Diabetic cardiac autonomic neuropathy is the damage of structure or functions of cardiac autonomic nerves due to chronic hyperglycaemia. In a research studying the therapeutic effect of electroacupuncture at Feishu (BL13), Xinshu (BL15), Yishu (EX-B3), Pishu (BL20), Neiguan (PC6), Quchi (LI11), Zusanli (ST36), and Sanyinjiao (SP6), the inhibition of NGF excessive expression was observed and the content of ChAT, CNTF, and GAP-43 raised strikingly, the latter three of which participate in the restoration of nervous injuries [36].

NGF has the biological functions of nutrition and growth promotion of nerves. It is essential in maintaining normal growth and development of nerves, but its excessive expression may lead to abnormal proliferation of neurons. As discussed above, we know that NGF has low expression in the patients with diabetic peripheral neuropathy, and their NGF increases after electroacupuncture. An experiment of rats has also proved that NGF can promote peripheral nerve regeneration [37]. But on the contrary, NGF is high in diabetic cardiac autonomic neuropathy which is caused by the endogenous NGF produced in myocardium for the maintenance of neural integrity in several heart tissues. This finding in a dog experiment also pointed out that exogenously infused NGF can also protect against neural stunning of sympathetic cardiac innervation [38]. And the electroacupuncture may have played the role of exogenous NGF in depressing the excessive expression of endogenous NGF. The specific 
mechanisms of the effect of electroacupuncture treatment on this disease need to be further studied.

3.3. Diabetic Foot. A diabetic foot exhibits ulcers or gangrenes resulted from lower limb microcirculation disorder led by diabetic neuropathy and vascular structural damage. 6-Keto- $\mathrm{PGF}_{1 \alpha}$ and thromboxane $\mathrm{B}_{2}\left(\mathrm{TXB}_{2}\right)$ are one of the most common and effective biochemical factors of regulating thrombosis balance, and pathological changes might break the balance and cause various disorders of blood circulation. Researchers discovered that Vitamin $\mathrm{B}_{1}$ injection at point Jiexi (ST41) increases the content of 6-Keto-PGF ${ }_{1 \alpha}$ and decreases that of $\mathrm{TXB}_{2}$ to maintain the original balance in grade 0 diabetic foot patients. They reckoned that the acupoint injection boosts the nutrition supplement of neurons, restores part of its functions and exert feedback influence on blood circulation. In the progress, specific regulating factors of blood circulation like 6-Keto-PGF $1 \alpha$ and $\mathrm{TXB}_{2}$ are affected and regional microcirculation is ameliorated [39].

3.4. Diabetic Anterior Horn Injury of Spinal Cord. Diabetic anterior horn injury of spinal cord is another common nervous lesion. It usually implicates motor neurons of spinal cord, etc., and brings about muscular atrophy and a decline of muscular tension. A study on diabetic model rats demonstrated that the protein expression of apoptosis gene of anterior horn neurons of spinal cord, Bax, was remarkably lowered while its correspondent antiapoptosis gene $\mathrm{Bcl}-2$ had a substantial increase after the treatment of electroacupuncture at Zusanli (ST36) and Yishu (EX-B3). As a result, the ratio of $\mathrm{Bcl}-2$ and $\mathrm{Bax}$ was upregulated and was approaching normal standards [40]. This suggests that electroacupuncture can modulate gene expression and prevent the apoptosis of neurons.

3.5. Diabetic Myopathy. As mentioned above, neuropathy can lead to myopathy, a complication of great importance but often neglected. The mechanism of low-frequency electrical stimulation at Yanglingquan (GB34) and Zusanli (ST36) involves a variety of biochemical signals. First, it upregulates IGF-1 signaling pathway and the expression of microRNA myomiR which is related to muscle regeneration. This is followed by an increase in phosphorylation level of factors concerning protein synthesis like AKT, FoxO1, mTOR, and p70S6. Then, a series of pathological effects in diabetic model rats are reversed including the reduction of Pax7, MyoD, myogenin, and MHC-emb expression. From the macroscopic view, the ramifications above can be embodied as promoting muscle regeneration, strengthening muscular functions and attenuating myopathy [41].

To summarize, the study of some of nervous disease is very limited. For instance, only grade 0 diabetic foot was studied in the category of diabetic foot but that of far severer grade still have urgent needs of research. Nevertheless, the mechanisms of different kind of neuropathy are concentrated on gene, mRNA, or protein regulation of neuron growth or apoptosis. Future studies can draw on ideas and experiences of similar nervous diseases and enrich the theories.

\section{The Mechanism Study of Diabetic Nephropathy Acupoint Treatment}

Diabetic Nephropathy (DN) is glomerular sclerosis led by glucose metabolism disorders, accompanied by pathognomonic symptoms like abnormal urinal protein. DN is one of the most common microvascular complications of diabetes, with an incidence of $20 \%-40 \%$ [42]. It is also one of the major causes of death for diabetic patients. Diabetic nephropathy is reversible at early stages, so prompt therapy may help injured kidney recover. We collected the mechanism study of acupoint injection, catgut implantation combined with Chinese medicine, and "spleen-stomach harmonizing" needling. These therapeutic means affect the structure and functions of kidney through different chemical signals.

Shuxuening injection at Zusanli (ST36), Sanyinjiao (SP6), Fenglong (ST40), Yinlingquan (SP9), and Zhongshu (DU7) alternately of DN patients is proved to have certain curative effects. Shuxuening is an extract from ginkgo leaf, which contains flavanoids, phenols, alkaloids, etc. The injection significantly decreases the expression of vascular endothelial growth factor (VEGF), the rise of which will cause an increase in expansion of extracellular matrix and mesangial matrix of kidney, and therefore lead to glomerular sclerosis [43]. Catgut implantation at Shenshu (BL23), Huiyang (BL35), and Guanyuan (BL26) combined with taking TCM prescription "Tangtong drink" cuts down the content of TGF- $\beta 1$ and IGF-1 in the serum of DN model rats at early stages [44]. The former one is the crucial cell factor in the mechanism of glomerular sclerosis while the latter participates in the alteration of glomerular hemodynamics, cell hypertrophy, etc. The therapies both downregulate the harmful growth of particular cell factors and promote the repair of renal structure. The possible ramifications of the regulation are the amelioration of glucose metabolism and renal hemodynamics, the correction of high filtration and high perfusion status of kidney, the reduction of growth and differentiation of renal cells, etc., all of which alleviate nephropathy and improve renal functions.

"Spleen-stomach harmonizing" needling also plays an important role in the clinical treatment of DN. Acupuncture at Zhongwan (CV12), Quchi (LI11), Hegu (LI4), Xuehai (SP10), Zusanli (ST36), and Yinlingquan (SP9) with this technique efficaciously improves glomerular filtration and reduce urinary albumin excretion rate of DN patients. Specialized study of it also found extensive regulation of the patients' bodies. During their study, a number of genes and signaling pathways were investigated. The study mentioned 5 signaling ways that are upregulated, including glutathione metabolism, the interactions of cell factor receptors, etc. The downregulated genes control altogether 40 signaling pathways, concerning type 1 diabetes mellitus, PPAR, etc. Among them, gene IFNG and the signaling pathways it regulates concerning $\mathrm{T}$ cell receptor (TCR), hypoxia inducible factor-1 (HIF-1), JAK-STAT signaling pathway, TGF- $\beta$, etc. stand out. Furthermore, spleen-stomach harmonizing needling inhibits the excessive expression of MCP-1. The combination of these effects modulates the amount and activity of $\mathrm{T}$ cell subset, thus restoring lymphocyte injury $[45,46]$. Meanwhile, the needling decreases the oxidative stress level of DN patients 
and strengthens the capability of organism to scavenge free radicals [47]. All of the above delay or alleviate renal damage.

\section{The Mechanism Study of Diabetic Hepatopathy Treatment}

Liver is of great significance to glucose metabolism of human body. Long-term hyperglycaemia may harm the structure of liver, thus counter-reflecting on glucose metabolism and forming a vicious cycle. As diabetic hepatopathy has only been raising people's awareness in recent years, the mechanism study of its treatment is still limited. The theories at present indicate that electroacupuncture at Yishu (EX-B3) enhances the expression of gene and protein of glucagonlike peptide-1 (GLP-1) receptor, a glucose-dependent insulin secretion enhancer, in the liver cells as well as decreases alanine aminatransferase (ALT) and aspartate aminotransferase (AST), and the biomarkers of liver cell damage, in the serum $[48,49]$. These alterations protect liver cells and ultimately ameliorate diabetic hepatopathy.

\section{Conclusions}

As the circumstance of diabetes grows increasingly severe all over the world, acupoint therapy, as one of the most important parts of Chinese medicine, plays an indispensable role in the treatment of diabetes and its complications.

We listed the function and effect of acupoint therapy on $\mathrm{DM}$ and its common chronic complications in Table 1. From what we have collected, the mechanism study of treatment of hyperglycaemia and its common chronic complications like peripheral neuropathy and nephropathy is comparatively elaborate. However, the correspondent studies of diabetic foot, diabetic anterior horn injury of spinal cord, diabetic myopathy, diabetic hepatopathy, and other complications unmentioned above like diabetic retinopathy are shallow and rare. For instance, only grade 0 diabetic foot has been studied in the category of diabetic foot. The most studied methods of acupoint therapy are electroacupuncture, acupoint injection, catgut implantation, and manual acupuncture (usually with "spleen-stomach harmonizing" needling technique), but the mechanism of other effective ways like pressing ear with beans, auricular points plaster therapy, and external application remains unclear. As shown in Figure 1, the most studied acupoints are Zusanli (ST36), which is mentioned 18 times in all the articles we collected. This is followed by Shenshu (BL23) (7 times), Sanyinjiao (SP6) (7 times), Yishu (EX-B3) (4 times), Zhongwan (CV12) (4 times), and Quchi (LI11) (4 times). The rest of the acupoints are widely distributed. Still, the effects of different acupoints as well as different combination of them need more investigations and experiments. Moreover, most of the studies are experiments on model rats rather than clinical trials, so whether the results are consistent with human body awaits further research. Whether electroacupuncture can substitute manual acupuncture is another problem unsolved hitherto.

The mechanisms concluded involve the regulation of nerve conduction, signal pathways, hormone level, protein expression, oxidative stress level, and structure restoration.

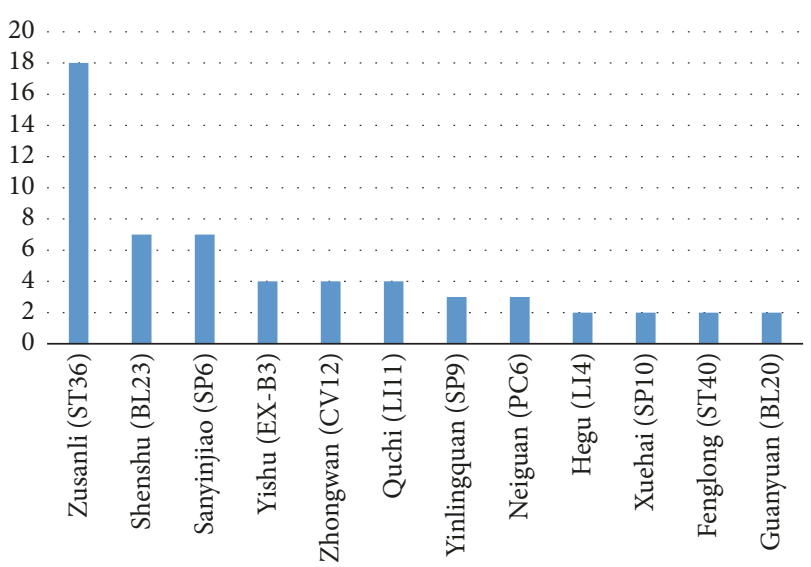

FIGURE 1: The appearance frequency of most mentioned acupoints in the articles of recent five years.

In biology, structures and functions are united and the abnormality of functions is usually concerned with structural damage. The treatment generally regulates some specific biochemical factors to influence particular signal pathways, eventually controlling the apoptosis and proliferation of cells and repairing the damaged structure. Sometimes the therapy has two-way regulation of one specific factor in different studies, and we speculate that the therapy is a process of balancing the whole body. Different pathology may cause rise or decline of the content of specific factors, and the therapy corrects them to go to the normal status. The studies usually focused on a few factors each time, but the therapy actually regulates the entire body. The nervous system, the endocrine system, the circulation system, etc. collaborate to achieve a more harmonious balance in human body. Due to the confined studies, it is basically impossible to draw a complete picture of the mechanism currently. Nevertheless, the present researches have laid the foundation and pointed out the possible directions. Future studies can draw on the experimental design and consideration of past researches, enrich the existing theories, and explore more on the clinical trials.

\section{Conflicts of Interest}

The authors declare that there are no conflicts of interest regarding the publication of this paper.

\section{Authors' Contributions}

Yiyi Feng and Yuchen Fang wrote the whole manuscript text which was revised by Yiming Hao. Yiming Hao and Yiqin Wang helped in the ideas of study. All authors (Yiyi Feng, Yuchen Fang, Yiqin Wang, and Yiming Hao) reviewed the manuscript. Yiyi Feng and Yuchen Fang contributed equally to this study and share first authorship.

\section{Acknowledgments}

This work was supported by Shanghai Municipal Commission of Health and Family Planning Project (no. 20164Y0279), 


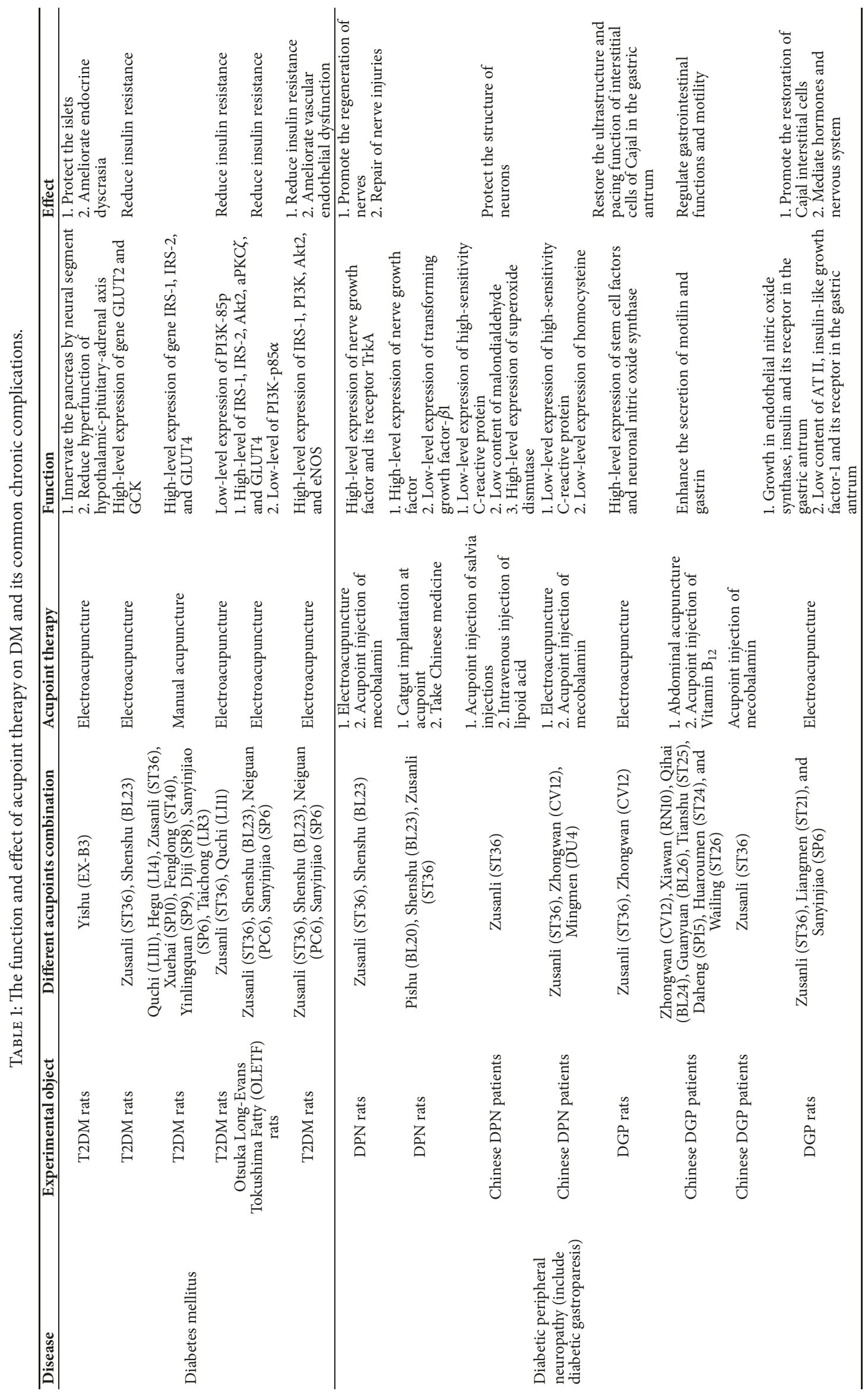




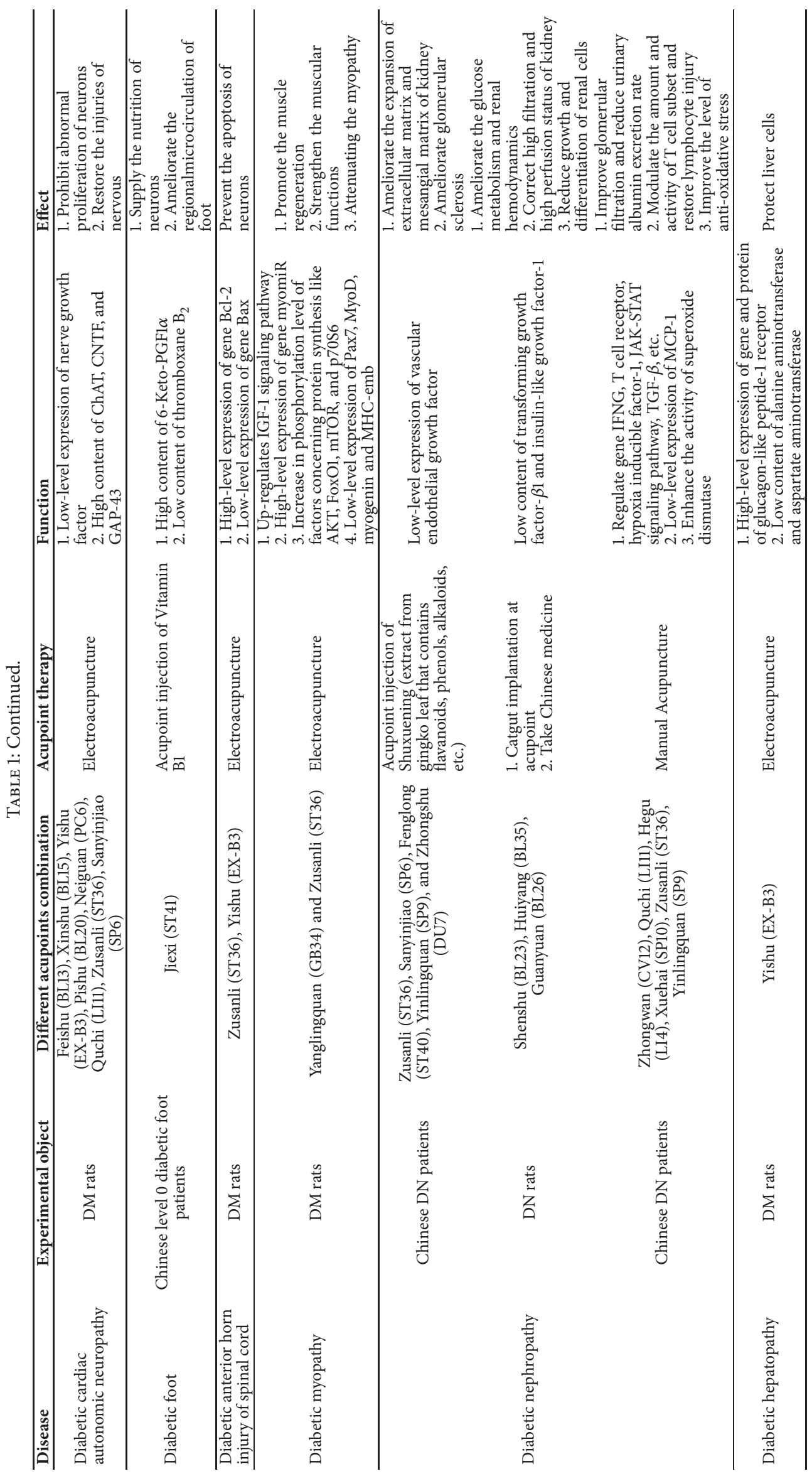


School Sports Scientific Research Project of Shanghai Municipal Education Commission (no. HJTY-2018-D12), National Basic Science Talent Training Fund Project (no. J1103607), and Shanghai Municipal College Students Innovation Project (no. 2018KCZ17).

\section{References}

[1] World Health Organization, “Global report on diabetes," 2016, http://www.who.int/diabetes/global-report/zh/.

[2] J. M. Garcia-Vivas, G. H. Carlos, B. C. Flavia et al., "Acupoint catgut embedding therapy with moxibustion reduces the risk of diabetes in obese women," Journal of Research in Medical Sciences the Official Journal of Isfahan University of Medical Sciences, vol. 19, no. 7, pp. 610-616, 2014.

[3] X. G. Hu, R. N. Li, N. Jia et al., "Electro-acupuncture on T2DM rats with points at different nerve segment," Journal of Clinical Acupuncture and Moxibustion, vol. 33, no. 6, pp. 57-60, 2017.

[4] P. Wang and S. Wang, "Study on the regularity of selecting acupoints according to the theory of inneruation of neucral segment," Acupuncture Research, 1998.

[5] S. Gao, R. Li, H.-H. Tian, E.-S. Pei, B.-Y. Cao, and Y. Wu, "Effects of electroacupuncture at 'Yishu' (EX-B 3) on the relative hormones of HPA axis in rats with type-2 diabetes mellitus," Chinese Acupuncture and Moxibustion, vol. 34, no. 11, pp. 10991105, 2014.

[6] N. Jia, R. B. Y. Li, and Cao., "Effects of electroacupuncture at "Zusanli" and "Shenshu" on GLUT2 and GCK in T2DM rats," World Chinese Medicine, vol. 12, no. 5, pp. 1114-1119, 2017.

[7] H. Chen, Z. L. Zhang, and X. Wang, "Effect of spleen-stomach harmonizing needling on insulin resistance and expression of insulin receptor substrate-1, -2 , and glucose transporter- 4 in insulin resistance type 2 diabetes rats," Acupuncture Research, vol. 42, no. 3, Article ID 197, 2017.

[8] X. Liu, J. F. He, Y. T. Qu et al., "Effects of electroacupuncture on insulin resistance and hypothalamic insulin signal molecule in rats with diet-induced obesity," Chinese Acupuncture \& Moxibustion, vol. 36, no. 9, pp. 957-961, 2016.

[9] Xin-Yu Huang, Liang Zhang, Jian Sun, Neng-Gu Xu I, and We Yi I, "Acupuncture Alters Expression of Insulin Signaling Related Molecules and Improves Insulin Resistance in OLETF Rats," Evidence-Based Complementary and Alternative Medicine, vol. 2016, Article ID 9651592, 7 pages, 2016.

[10] Y. K. Lu, Effects of acupuncture on oxidative stress on OLETF rats [Doctoral, thesis], Guangzhou University of Chinese Medicine, 2015.

[11] D. Lan, N. Xu, J. Sun et al., "Electroacupuncture mitigates endothelial dysfunction via effects on the PI3K/Akt signalling pathway in high fat diet-induced insulin-resistant rats," Acupuncture in Medicine, vol. 36, no. 3, pp. 162-169, 2018.

[12] S. X. Hu, X. Lu, Z. L. Chen et al., "Research progress on the acupuncture treatment of diabetic peripheral neuropathy," Chinese Journal of Acupuncture and Moxibustion, vol. 3, pp. 1922, 2013.

[13] V. Bril, "Neuromuscular complications of diabetes mellitus," Continuum: Lifelong Learning in Neurology, vol. 20, no. 3, pp. 531-544, 2014.

[14] M. Mehra, S. Merchant, S. Gupta, and R. C. Potluri, "Diabetic peripheral neuropathy: Resource utilization and burden of illness," Journal of Medical Economics, vol. 17, no. 9, pp. 637-645, 2014.
[15] Q. Dong, W. P. Cao, and J. Lu, "Comparative study of protective effect of electroacupuncture and acupoint injection on the nerves of diabetic peripheral neuropathy model rats. Lishizhen Medicine and Material Medical research," Lishizhen Medicine and Material Medical research, vol. 24, no. 4, pp. 1001-1003, 2013.

[16] L. Qu, Effect of catgut embedding therapy and Tangtong drink on NGF and TGF- $\beta 1$ level of diabetic neuropathy rats [Master, thesis], Guizhou Medical University, 2017.

[17] W. J. Li, "Effect of acupoint injection combined with lipoic acid on MDA, SOD, hs-CRP and subjective symptoms of diabetic peripheral neuropathy," Journal of Military Surgeon in Southwest China, vol. 2, pp. 159-161, 2015.

[18] Y. X. Li, X. F. Liu, and Wan., "Study on the mechanism of acupuncture at points of urinary bladder meridian in the treatment of diabetic peripheral neuropathy," Journal of Clinical Acupuncture and Moxibustion, vol. 5, pp. 16-18, 2015.

[19] D. C. Yin, M. Chen, X. N. Wang et al., "Effects of diabetes treatment instrument combined with mecobalamin on diabetic peripheral neuropathy and its influence on the level of Hs-CRP and Hcy," Journal of Hunan Normal University (Medical Science), vol. 4, pp. 21-24, 2014.

[20] G. Wei, H. Q. Li, Z. N. Yang et al., "Curative effect of abdominal acupuncture combined with acupoint injection on motilin and gastrin levels of diabetic gastroparesis," Journal of Clinical Acupuncture and Moxibustion, vol. 32, no. 3, pp. 1-4, 2016.

[21] Y. Gao, G. Y. Y. Lu, and Wang., "Prospects and progress on the mechanisms of acupuncture underlying improvement in diabetic gastroparesis," Acupuncture Research, vol. 42, no. 4, pp. 367-371, 2017.

[22] C. C. Zhang, Y. P. Lin, and Y. Peng, "Effects of electroacupuncture on ultrastructure of interstitial cells of Cajal and stem cell factor-kit signal pathway of gastric antrum in diabetic gastroparesis rats," Acupuncture Research, vol. 42, no. 6, 2017.

[23] C. Zhang, Y. Lin, Y. Peng et al., "Study on the mechanisms of electroacupuncture for promoting gastrointestinal motility in rats with diabetic gastroparesis," Journal of Acupuncture and Tuina Science, vol. 15, no. 3, pp. 158-164, 2017.

[24] H. Chen, W. Zhu, J. Lu et al., "The effects of auricular electroacupuncture on ameliorating the dysfunction of interstitial cells of cajal networks and nNOSmRNA expression in antrum of STZ-induced diabetic rats," PLoS ONE, vol. 11, no. 12, Article ID e0166638, 2016.

[25] Y. Chen, J. J. Xu, S. Liu, and X. H. Hou, "Electroacupuncture at ST36 ameliorates gastric emptying and rescues networks of interstitial cells of Cajal in the stomach of diabetic rats," PLoS ONE, vol. 8, no. 12, article e83904, 2013.

[26] L. Tian, B. Zhu, and S. Liu, "Electroacupuncture at ST36 protects ICC networks via $\mathrm{mSCF} / \mathrm{Kit}-\mathrm{ETV} 1$ signaling in the stomach of diabetic mice," Evidence-Based Complementary and Alternative Medicine, vol. 2017, Article ID 3980870, 13 pages, 2017.

[27] Y. Chen and X. Ye., "Effect of acupoint injection combined with mosapride on gastric emptying time and gastrointestinal hormone level of diabetic gastroparesis," Guang Ming Zhong Yi=Journal of Traditional Chinese Medicine, vol. 12, pp. 26282630, 2015.

[28] L. Liu, X. F. Wu, and X. N. Zheng, "Effect of point-moxibustion and electroacupuncture on the expression of endothelial nitric oxide synthase mRNA and angiotensin 2 mRNA in gastric antrum in diabetic gastroparesis rats," Acupuncture Research, vol. 42, no. 3, pp. 240-245, 2017. 
[29] J. W. Yang, Y. P. Lin, and H. J. Chen, "Effects of electroacupuncture on the expression of INS and IR in gastric antrum of diabetic gastroparesis rats," Journal of Hunan University of Chinese Medicine, vol. 37, no. 2, pp. 172-176, 2017.

[30] J. W. Yang, Y. Peng, and H. J. Chen, "Effect of electroacupuncture intervention on gastrointestinal motility and expression of insulin-like growth factor 1 and its receptor proteins in gastric antrum in diabetic gastroparesis rats," Acupuncture Research, vol. 42, no. 4, pp. 315-320, 2017.

[31] Y. Q. Li, B. T. Yu, and Li., "Effects of acupuncture at single acupoint and combined acupoints on antral interstitial cells of Cajal in diabetic gastroparesis model rats," World Chinese Medicine, vol. 11, no. 2, pp. 214-218, 2016.

[32] Y. P. Lin, F. E. He, Y. Peng et al., "Effects of electroacupuncture on gastrointestinal hormones in rats with diabetic gastroparesis," Journal of Beijing University of Traditional Chinese Medicine, vol. 38, no. 12, pp. 847-851, 2015.

[33] Y.-P. Lin, Q.-Q. Wan, Y. Peng, F.-E. He, and J. Shen, "Effect of electroacupuncture at "Zusanli" (ST 36), etc. On gastrointestinal motility and expression of ghrelin mRNA and growth hormone secretagogue receptor mRNA in diabetic gastroparesis rats," Acupuncture Research, vol. 40, no. 4, pp. 290-295, 2015.

[34] Y. Peng, Y.-P. Lin, F.-E. He et al., "Effect of electroacupuncture on gastric motility, expressions of ghrelin and GHSR mRNA in gastric antrum tissue of diabetic gastroparesis rats," Journal of Acupuncture and Tuina Science, vol. 15, no. 2, pp. 88-93, 2017.

[35] H.-J. Chen, Y.-P. Lin, J.-W. Yang et al., "Effect of electroacupuncture on electrogastrogram and gastric antrum ghrelin in rats with diabetic gastroparesis," Journal of Acupuncture and Tuina Science, vol. 15, no. 4, pp. 242-249, 2017.

[36] E. Z. Zhu, N. X. Li, and Y. Zhang, "Regulating effect of electroacupuncture in the right antrium autonomic nerve damage of type 2 diabetic rats," Journal of Changchun University of Chinese Medicine, vol. 33, no. 6, pp. 893-896, 2017.

[37] W. Sun, C. Sun, H. Lin et al., "The effect of collagen-binding NGF- $\beta$ on the promotion of sciatic nerve regeneration in a rat sciatic nerve crush injury model," Biomaterials, vol. 30 , no. 27 , pp. 4649-4656, 2009.

[38] T. Abe, D. A. Morgan, and D. D. Gutterman, "Protective role of nerve growth factor against postischemic dysfunction of sympathetic coronary innervation," Circulation, vol. 95, no. 1, pp. 213-220, 1997.

[39] H. W. Song, B. L. Yu, H. W. Zhou et al., "Effect of Jiexi (ST41) acupoint injection of vitamin B1 on 6-Keto-prostaglandin(6Keto-PF1a) and thromboxane B2(TXB2) of level 0 diabetic foot patients," Journal of Clinical Acupuncture and Moxibustion, vol. 32, no. 1, pp. 32-34, 2016.

[40] W. Wang, F. J. Wu, and Zhao., "Effects of electroacupuncture on $\mathrm{Bax}$ and $\mathrm{Bcl}-2$ protein expression in anterior horn of spinal cord in diabetic rat models," Journal of Youjiang Medical University for Nationalities, vol. 38, no. 3, pp. 250-253, 2016.

[41] Z. Su, A. Robinson, L. Hu et al., "Acupuncture plus lowfrequency electrical stimulation (Acu-LFES) attenuates diabetic myopathy by enhancing muscle regeneration," PLOS ONE, vol. 10, no. 7, Article ID e0134511, 2015.

[42] H. Y. Zeng, Y. Cao, Y. Zhao et al., "Correlative analysis between the related indexes of obesity and the morbidity of type 2 diabetic nephropathy," World Journal of Integrated Traditional and Western Medicine, vol. 9, no. 6, Article ID 217, 2014.

[43] L. Song, Z. X. Dong, and S. Liang, "Study on the effect of acupoint injection on the expression of VEGF of diabetic nephropathy patients," For all Health, vol. 11, no. 4, 2017.
[44] Z. M. Guan, X. H. Bo, and L. Qu, "Effect of catgut embedding therapy and Tangtong drink on TGF- $\beta 1$ and IGF-1 level of early diabetic nephropathy rats," Journal of Guiyang Medical College, vol. 40, no. 12, pp. 1352-1355, 2015.

[45] Z. L. Zhang, S. H. Zhao, and X. Li, "Clinical obeservation on repair of lymphocyte injury in patients with diabetic nephropathy treated by regulating spleen-stomach needling," Chinese Acupuncture and Moxibustion, vol. 33, no. 12, pp. 1065-1070, 2013.

[46] X. Lu, The mechanism study of needling method of harmonizing spleen-stomach on impairment and repairment of lymphocytes in the patients with diabetic nephropathy [Doctoral, thesis], Tianjin University of Traditional Chinese Medicine, 2015.

[47] M. Wang, Z. L. Zhang, X. Q. Ji et al., "Ramdomized controlled clinical trial for analyzing effect of, spleen-stomach regulation needling, on oxidative stress level in patients with diabetic nephropathy," Acupuncture Research, vol. 40, no. 5, pp. 409-414, 2015.

[48] Y. Y. Wang, Study on the effect of electroacupuncture on, Yishu , (EX-B3) point on liver injury and GLP-1 receptor in rats with type 2 diabetic mellitus [Master, thesis], Beijing University of Traditional Chinese Medicine, 2017.

[49] Y. Y. Wang, R. Li, and H. H. Tian, "Effect of electroacupuncture on, Yishu, (EX-B3) point on post-liver injury transaminases in rats with type 2 diabetic mellitus," Journal of Clinical Acupuncture and Moxibustion, vol. 33, no. 2, pp. 41-45, 2017. 


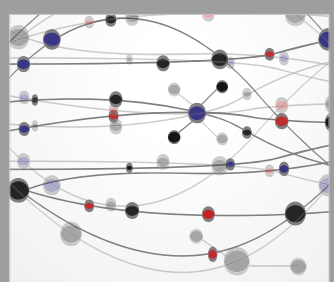

The Scientific World Journal
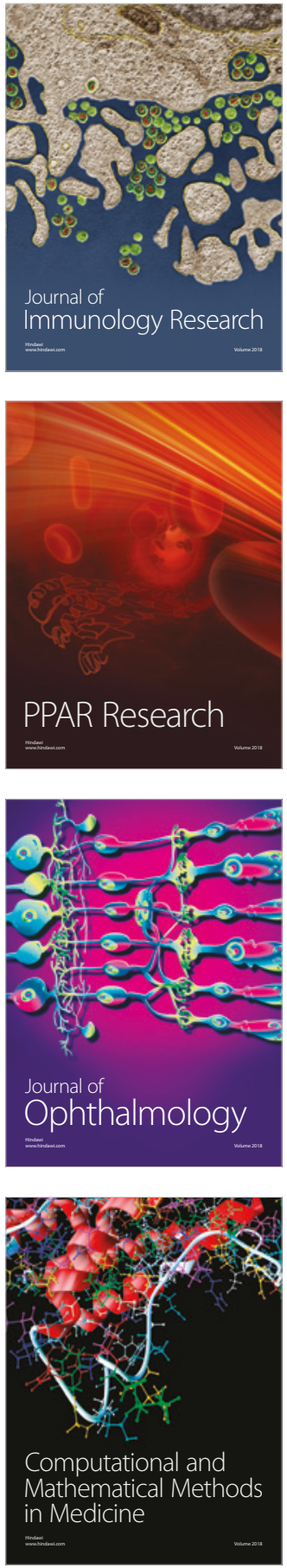

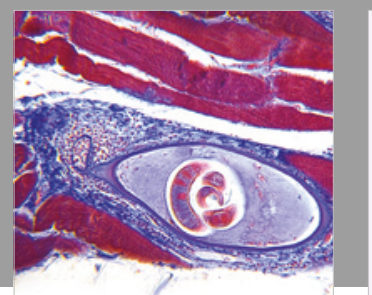

Gastroenterology Research and Practice

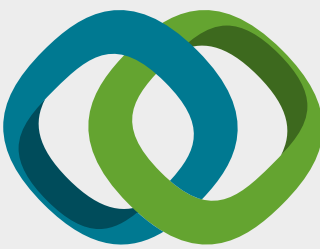

\section{Hindawi}

Submit your manuscripts at

www.hindawi.com
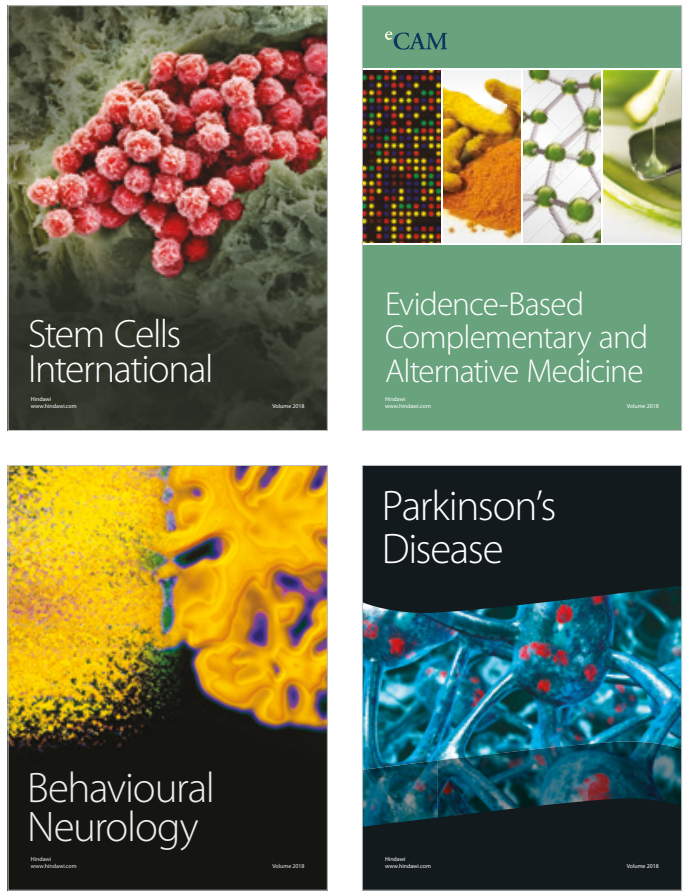

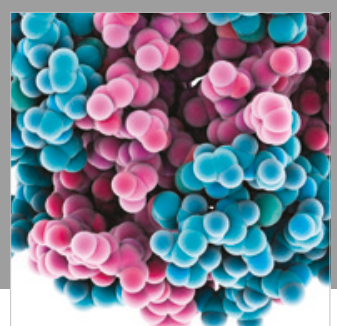

ournal of

Diabetes Research

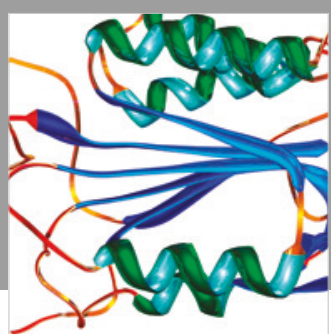

Disease Markers
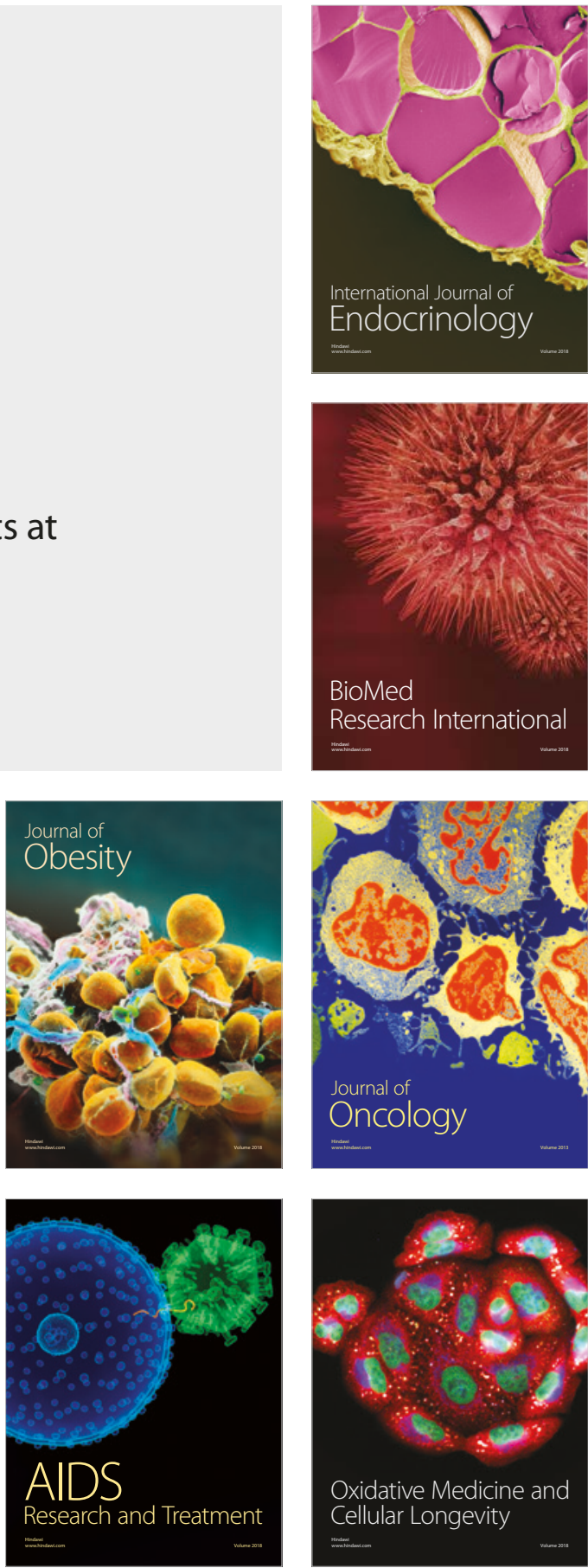